Das Acetylammoniumoxydhydrat : $\left.\underset{\mathrm{H}_{3}}{\mathrm{C}_{4} \mathrm{H}_{3}}\right\}$ No, $\mathrm{HO}=$ $\mathrm{C}_{4} \mathrm{H}_{7} \mathrm{NO}_{2}$ ist mit Aldehyd-Ammoniak $\mathrm{C}_{4} \mathrm{H}_{3} \mathrm{O}, \mathrm{HO}^{\sim} \mathrm{H}_{3} \mathrm{~N}=$ $\mathrm{C}_{4} \mathrm{H}_{7} \mathrm{NO}_{2}$ isomer, und während es einige Eigenschaften mit demselben gemein hat, wie z. B. die Reaction gegen Silbersalze, zeichnet es sich durch seine Nichtllüchtigkeit, seine basischen Eigenschaften und die Besländigkeit gegen Säuren und Alkalien aus. Alle diese Unterschiede finden in der Verschiedenheit der rationellen Formeln beider Verbindungen ihre vollkommene Erklärung.

Ich bin jetzt mit der weiteren Durchführung der Untersuchung in dem oben angedeuteten Sinne beschäfligt.

Untersuchung einiger Verbindungen des SenfölSchwefelwasserstoffs ;

von Heinrich Will.

In meiner Abhandlung über das ätherische Oel des schwarzen Senfs ${ }^{*}$ ) habe ich die Existenz einer Verbindung von Senföl mit Schwefelwasserstoff, von der Formel $\mathrm{C}_{8} \mathrm{H}_{8} \mathrm{NS}_{2}$, $\mathrm{H}_{2} \mathrm{~S}_{2}$ nachgewiesen. Diese Verbindung ist von L. Gmelin ${ }^{* *}$ ) Schwefelsenfsäure genannt worden ; Gerhard $t * * *$ ) betrachtet sie als Sulfocarbaminsäure, in welcher ein Aequivalent Wasserstoff durch das Radical Allyl ersetzt ist :

*) Diese Annalen, LII, 30.

**) Handb. d. Chemie, 4. Aufl, V. 207.

***) Traitè de Chimie org. II, 403. 
Sulfocarbaminshüu

$\mathrm{NH}_{2}(\mathrm{CO})_{2} \cdot \mathrm{S}$ )

$$
\text { H. S }
$$

Allylsulfocarbaminsäure

$\left.\mathrm{NH}\left(\mathrm{C}_{6} \mathrm{H}_{3}\right)(\mathrm{CS})_{2} \cdot \mathrm{S}\right\}$

Nach der von mir dafür aufgestellten Formel wäre sie Schwefelcyanallyl, verbunden mit Schwefelwasserstoff :

$$
\left.\left.\underset{\mathrm{C}_{6} \mathrm{H}_{5}}{\mathrm{C}_{2} \mathrm{~N}}\right\} \mathrm{~S}_{2}+{ }_{\mathrm{H}}^{\mathrm{H}}\right\} \mathrm{S}_{2} \text {. }
$$

Ich erhielt die Verbindung früher durch Einwirkung einer alkoholischen Kalilösung auf Senföl, wo sie, neben Kohlensäure und dem ölartigen Körper $\mathrm{C}_{14} \mathrm{H}_{14} \mathrm{~N}_{2} \mathrm{~S}_{2} \mathrm{O}_{2}$, nach der Gleichung

$$
\left.\begin{array}{r}
3 \mathrm{C}_{8} \mathrm{H}_{5} \mathrm{NS}_{2} \\
\mathrm{H}_{6} \mathrm{O}_{6}
\end{array}\right\}=\left\{\begin{array}{l}
\mathrm{C}_{8} \mathrm{H}_{7} \mathrm{NS}_{4} \\
\mathrm{C}_{14} \mathrm{H}_{14} \mathrm{~N}_{2} \mathrm{~S}_{4} \mathrm{O}_{2} \\
\mathrm{C}_{2} \mathrm{O}_{4}
\end{array}\right.
$$

entsteht. Aus der stark mit Wasser verdünnten Lösung wird, nach Abscheidung des ölartigen Körpers und Neutralisiren mit Essigsüure, durch essigsaures Bleioxyd ein citrongelbes, leicht veränderliches Bleisalz gefällt, dessen Analyse zur Formel $\left.\mathrm{C}_{8} \mathrm{H}_{3} \mathrm{NS}_{2}+\underset{\mathrm{PbS}}{\mathrm{HS}}\right\}$ führle.

Es läfst sich, wie ich gefunden habe, mit Leichligkeit die ganze Menge des Senföls in diese Sulfosäure überführen, wenn man dasselbe direcl mit Auflösungen von Schwefelwasserstoff-Schwefelmelallen in Alkohol zusammenbringt. Die Analyse der nachstehend beschriebenen und in dieser Weise dargestellten Verbindungen zeigl, dafs die früher von mir aus dem Bleisalz abgeleitete Zusammenselzung die richlige ist.

\section{Senföl-Schwefelwasserstoff-Schwefelanmonium :}

$$
\left.\left.\mathrm{C}_{8} \mathrm{H}_{10} \mathrm{~N}_{2} \mathrm{~S}_{4}=\underset{\mathrm{C}_{6}}{\mathrm{C}_{2} \mathrm{~N}}\right\} \mathrm{~S}_{2}+\underset{\mathrm{NH}_{4}}{\mathrm{H}}\right\} \mathrm{S}_{2} \text {. }
$$

Tröpfelt man Senföl in eine gesälligte und farblose Lösung von Schwefclwasserstoff - Schwcfelammonium in starkem Alkohol, so verschwindet der Geruch des ersteren augenblicklich; die Flïssigkeit erhitzt sich stark und nach einigen 
Augenblicken erstarrt sie zu einem Brei von farblosen Blättchen des obigen Salzes.

Zur Analyse wurde die in leeren Raum getrocknete Substanz verwendet.

I. 0,4463 gaben 0,520 Kohlensäure und 0,2768 Wasser. $0,617,1,705$ Platinsalmiak.

$0,5252 " 1,6403$ schwefelsauren Baryt.

II. $0,4385 \gg 0,5053$ Kohlensäure und 0,2675 Wasser. $0,659 \quad 2,069$ schwefelsauren Baryt.

Diefs entspricht in 100 Theilen :

I.

Kohlenstoff $31,70 \quad 31,36$

Wasserstoff $\quad 6,90 \quad 6,77$

Sticksiof $\quad 17,40$

Schwefel $\frac{43,03}{99,03 .}$
II.

43,30

Die obige Formel verlangt :

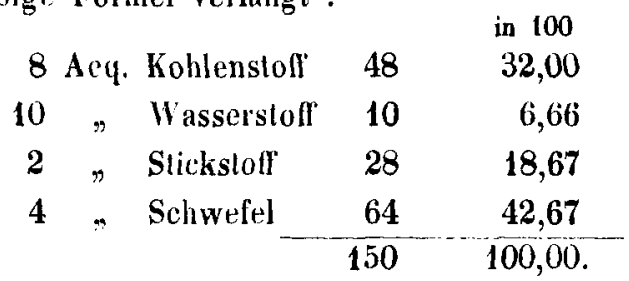

Das Salz ist nur wenig beständig; es zersetzt sich, ähnlich den folgenden Verbindungen, beim Aufbewahren.

$$
\begin{aligned}
& \text { Senfol-Schwefelwasserstoff-Schwefelkalium : } \\
& \left.\left.\qquad \mathrm{C}_{8} \mathrm{H}_{6} \mathrm{NS}_{4} \mathrm{~K}={ }_{\mathrm{C}_{6}}^{\mathrm{C}_{2} \mathrm{~N}} / \mathrm{H}_{5}\right) \mathrm{~S}_{2}+{ }_{\mathrm{K}}^{\mathrm{H}}\right\} \mathrm{S}_{1} .
\end{aligned}
$$

Eine alkoholische oder auch eine wässerige Lösung von Schwefelwasserstoff - Schwefelkalium, die man so lange mit Senföl versetzt hat, als der Geruch des letzteren noch verschwindet, liefert beim langsamen Verdunsten im leeren Raum und bei nicht zu unbeträchtlichen Mengen grofse 
rhombische Tafeln von oft zollgrolsem Durchmesser; beim rascheren Verdampfen bilden sich nadelförmige Krystalle. Sie sind in der Flüssigkeit durchsichtig und farblos; an der Luft werden sie undurchsichtig, gelb, unter Verlust ihrer Form und ihrer vollkommenen Löslichkeit in Wasser, welches alsdann eine zähe schwefelgelbe Masse hinterläfst. Die wässerige Lösung des frisch bereiteten Salzes läfst sich erhitzen, ohne den Geruch nach Senföl zu entwickeln; versetzt man die erwärmte Lösung mit salpetersaurem Silheroxyd, so tritt unter Fällung von Schwefelsilber sogleich der Geruch des Senföls auf; ebenso entwickelt die trockene Verbindung beim Erhitzen Senföl.

Von dem luftrockenen Salz gaben bei der Analyse 1,2630 Grm. 0,621 schwefelsaures Kali. Ferner lieferten 0,7825 Grm. 2,161 schwefelsauren Baryt.

Diefs entspricht in 100 Theilen :

$$
\begin{array}{ll}
\text { Kalium } & 22,50 \\
\text { Schwefel } & 38,09 .
\end{array}
$$

Die obige Formel verlangt :

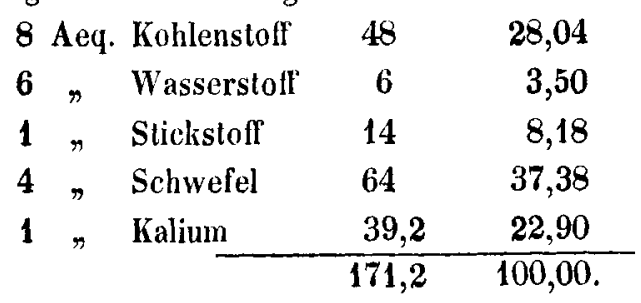

$$
\begin{aligned}
& \text { Senföl-Schroefelkalium : } \\
& \text { a. } \mathrm{C}_{8} \mathrm{H}_{3} \mathrm{NS}_{2}+2 \mathrm{KS} \text {. }
\end{aligned}
$$

Eine mil Senföl vermischte weingeistige Auflösung von Einfach-Schwefelkalium setzt, wenn letzteres in geringem Ueberschufs vorhanden ist, bein gelinden Verdunsten ein weifses, körniges Salz ab, welches beim Erhilzen, olıne seine Farbe zu ändern, Senföl entwickelt. 
0,5563 Grm. dieses Salzes gaben 0,483 Grm. schwefelsaures Kali. Hieraus in 100 Theilen :

$$
\begin{array}{ccc}
\text { Kalium } & 39,2 & \begin{array}{r}
\text { berechnet } \\
\mathrm{C}_{8} \mathrm{H}_{5} \mathrm{NS}_{4} \mathrm{~K}_{3} \\
37,4 .
\end{array} \\
\text { b. } & \mathrm{C}_{8} \mathrm{H}_{3} \mathrm{NS}_{2}+\mathrm{KS} .
\end{array}
$$

Die Multerlauge, aus der sich das vorhergehende Salz abgeschieden hat, liefert beim Stehen im leeren Raum über Schwefelsäure nadelförmige, kaum gelblich gefärbte lírystalle, welche sich dem vorigen Salz ähnlich verhalten, jedoch weniger Kalium enthalten.

I. 0,6270 gaben 0,356 schwefelsaures Kali.

II. $0,2684 \Rightarrow 0,1494 \quad$ n

Diefs entspricht in 100 Theilen :

\begin{tabular}{cccc} 
& \multicolumn{2}{c}{ gefunden } & berechnet \\
Kalium & I. & II. & $\mathrm{C}_{8} \mathrm{H}_{5} \mathrm{NS}$, KS \\
25,5 & 25,0 & $25,4$.
\end{tabular}

Das Senföl vereinigt sich demnach nicht blofs mit Schwefelwasserstoff-Schwefelmetallen zu krystallisirbaren Salzen, sondern auch mit löslichen Einfach-Schwefelmetallen, und zwar, wie es nach der vorstehenden Analyse scheint, in verschiedenen Verhältnissen.

$$
\begin{gathered}
\text { Senföl-Schwefelwasserstoff-Schwefelnatrium : } \\
\qquad \mathrm{C}_{8} \mathrm{H}_{5} \mathrm{NS}_{2}, \underset{\mathrm{H}}{\mathrm{Ha}} \mid \mathrm{S}_{2}+6 \mathrm{HO} .
\end{gathered}
$$

Diese Verbindung erhält man leicht beim Vermischen einer warmen weingeistigen Auflösung von Schwefelwasserstoff-Schwefelnatrium mit Senföl, so lange der Geruch des letzteren noch verschwindet. Sie scheidet sich in perlmutterglänzenden, fettig anzufühlenden Blättchen $a b$, die beim Erhitzen zuerst schmelzen und dann reichlich Senföl entwickeln. Das Salz enthält Krystallwasser und lälst sich so wenig wie die vorhergehenden ohne Zerselzung aufbewahren. 
I. 0,730 gaben 0,6345 Kohlensäure und 0,391 Wasser. 0,394 gaben 0,133 schwefelsaures Natron.

II. 0,6385 gaben 0,216 schwefelsaures Natron.

Diefs entspricht :

\begin{tabular}{|c|c|c|c|c|c|c|}
\hline \multirow[b]{2}{*}{8} & \multirow[b]{2}{*}{ Aeq. } & \multirow[b]{2}{*}{ Kohlenstoff } & \multicolumn{2}{|c|}{ Theorie } & \multicolumn{2}{|c|}{ Versuch } \\
\hline & & & 48 & 23,90 & 23,70 & - \\
\hline 12 & $\eta$ & Wasserstofl & 12 & 5,73 & 5,93 & - \\
\hline 1 & $n$ & Stickstoff & 14 & - & 一 & 一 \\
\hline 4 & $n$ & Schwefel & 64 & - & - & - \\
\hline 6 & $n$ & Sauerstoff & 48 & 一 & - & - \\
\hline 1 & » & Natrium & $\frac{23,1}{155,1}$ & 11,10 & 11,00 & 11,4 \\
\hline
\end{tabular}

$$
\begin{aligned}
& \text { Senföl-Schwefelwasserstoff-Schwefelbarium : } \\
& \qquad \mathrm{C}_{8} \mathrm{H}_{5} \mathrm{NS}_{2}, \underset{\mathrm{H}}{\mathrm{Ba} /} \mathrm{S}_{2}+4 \mathrm{HO} .
\end{aligned}
$$

Dieses Salz entsteht beim Erwärmen einer mit Schwefelwasserstoff übersätligten und mit etwas Weingeist versetzten Schwefelbariumlösung mil Senföl, oder wenn man Barythydrat und Senföl in Wasser vertheilt und unter Zusalz von Weingeist Schwefelwasserstoff einleitet; es setzt sich auch aus der Mutterlauge des in Weingeist schwerer löslichen Salzes ab, dessen Analyse nachstehend gegeben ist und welches Einfach-Schwefelbarium enthält. - Das sehr leicht lösliche Salz bildet der Natriumverbindung ähnliche Krystallblättchen.

I. 0,464 eines nach dem ersteren Verfahren bereitelen Salzes gaben 0,2405 schwefelsauren Baryt.

II. 0,6465 eines Salzes, welches aus der Mutterlange der durch Weingeist abgeschiedenen folgenden Bariumverbindung beim freiwilligen Verdunsten anschols, lieferten 0,965 schwefelsauren Baryt.

0,4985 desselben Salzes gaben zur Schwefelbestimmung mit Aelzkali und Salpeter geschmolzen 0,965 schwefelsauren Baryt. 
0,6988 gaben 0,583 Kohlensäure und 0,290 Wasser.

Diels führt zur obigen Formel. welche verlangt :

beredinet

\begin{tabular}{|c|c|c|c|c|c|c|}
\hline 8 & Aeq. & Kohlenst ofl" & 48 & 20,20 & I. & $\begin{array}{c}\text { II. } \\
\mathbf{1 9 , 9 0}\end{array}$ \\
\hline 10 & " & Wasserstoll & 10 & 4,23 & - & 4,60 \\
\hline 1 & , & Stickstofl & 14 & - & - & - \\
\hline 4 & - & Schwefel & 64 & 27,05 & - & 26,5 \\
\hline 4 & 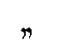 & Sauerstoff & 32 & - & - & - \\
\hline 1 & , & Barium & 68,6 & 29,00 & 30,4 & 30,44 \\
\hline
\end{tabular}

\section{Senföl-Schwefelbarium : \\ $\mathrm{C}_{8} \mathrm{H}_{5} \mathrm{NS}_{2}+2 \mathrm{BaS}+2 \mathrm{HO}$.}

Vermischt man gelbe, erwärmte Schwefelbariumlösung, so wie man sie durch Behandeln des rohen Schwefelbariums mil Wasser erhält, nach und nach mit senföl, bis der Germch des letzleren bleibend wird, und filtrirt, so erhält man beim Erkalten farblose oder schwach gelbliche Blattchen, die an der Luft zu einem weifsen Pulver zerfallen. Die nämliche Verhindung wird durch Weingeist ausgefällt. Das salz riecht nach Senföl, schmilzt in der Wärone nicht und verolimmt beim stiarkeren Erhitzen. Zur Analyse wurde das durch wiederholtes Pressen zwischen Papier getrocknete Salz angewendet.

I. 0,722 Grm. des aus der wässerigen Lösung krystallisirten Salzes gaben 0,6040 schwefelsauren Baryt.

II. 0,355 des durch Weingeist abgeschiedenen Salzes gaben 0,286 schwefelsaurou baryl, enlsprechend :

$$
\begin{gathered}
\text { gefunden } \\
\text { I. II. } \quad \mathrm{C}_{8} \mathrm{H}_{5} \mathrm{NS}_{2}, 2 \mathrm{BaS}, 2 \mathrm{HO}
\end{gathered}
$$$$
\text { Barimu 49,1 47,3 47,94. }
$$

Diese Verbindung scheint mit einem verschiedenen Wassergehalt krystallisiren zu kömen, sofern cin Salz einer 
anderen Breilung 41,6 pC. Barium bei der Analyse gab, was der Formel $\mathrm{C}_{8} \mathrm{H}_{5} \mathrm{NS}_{2}, 2 \mathrm{BnS}+6 \mathrm{HO}$ (hereclinel 42,6 $\mathrm{Ba}$ ) entsprechen wuirde.

\section{Senföl-Schwef elvoasserstoff - Schwefulrulcium.}

Vermisiht man Kalkmilch mit Senföl und, zur Lösung des letzteren, mit elwas Weingeist, so verschwindet beim Einleiten von sclıwefolwassersloff bis zur Ucbersültigung der Geruch vollkummet. Man erhailt eine klare Lissung, die nach dem Verdunsten im Wasserbade die Calciumverbindung als schivach gelblich gefärble, durchsichlige Grallerte hinterlälst. Beim vollkommenen Trocknen zerfälli sie unler reichlicher Lintwickelung von Senföl.

\section{Ueber den Cumin - Alkohol;}

von Dr. C. Kraut*).

Gerhardt und Cahours **) haben gezeigt, dafs das in samen von Cuminum Cyminum entbaltene flüchlige Oel in Handel unter dem Nanıen Römisch-Kümmelöl bekannı ein Gemenge zwcier Verbindungen ist, einer sauerstolffreien, des Cymens, und eintr sauersloflhaltigen, des Cuminols. Dieser letzlere liörpor ist dem Bittermandeliil homolog und steht in demsc!lıen Verhältnils zur Cuninsiuure, wie dieses zur Benzoissäure; das heil'st er ist der Aldehyd der Cuminsiure.

Wälırend das Cuminol durch sclmelzendes Kalihydrat wrnigstens gröfstentheils unler Wasserstoffentwickelung direct

*) Aus desse» Inaugural-IJissertation. liüttingen 1854.

**) Jin'se Aunalen X.XXVIII. 67. 